Nervenarzt 2014 • 85:135-136

DOI 10.1007/s00115-013-3868-7

Online publiziert: 6. Februar 2014

(c) Springer-Verlag Berlin Heidelberg 2014
T. Schläpfer ${ }^{1}$ · J. Volkmann ${ }^{2}$ - G. Deuschl ${ }^{3}$

${ }^{1}$ Klinik für Psychiatrie und Psychotherapie, Universitätsklinikum Bonn

2 Neurologische Klinik, Universitätsklinikum Würzburg

${ }^{3}$ Klinik für Neurologie, UKSH, Campus Kiel

\title{
Tiefe Hirnstimulation in Neurologie und Psychiatrie
}

Die tiefe Hirnstimulation (THS) ist ein Therapieverfahren, das zentrale neuronale Kreisläufe durch elektrische Stimulation moduliert und innerhalb des letzten Jahrzehnts die Behandlung einiger neurologischer Erkrankungen revolutioniert hat. Gegenwärtig wird die THS bei Bewegungsstörungen im Rahmen des Morbus Parkinson, bei essenziellem Tremor sowie bei Dystonien routinemäßig eingesetzt. Seit einigen Jahren wird die THS auch systematisch als potenzielles Behandlungsverfahren bei einigen therapieresistenten psychiatrischen Erkrankungen untersucht. Diese neuen psychiatrischen Anwendungsmöglichkeiten profitieren in Bezug auf ihre wissenschaftliche Entwicklung enorm von den Erfahrungen, die vorher bei neurologischen Indikationen gemacht wurden.

\section{》) THS wird Teil des \\ selbstverständlichen \\ Behandlungsspektrums werden}

Die Rationale der THS beruht auf der zunehmenden Erkenntnis, dass eine Reihe neurologischer oder psychiatrischer Symptombildungen auf Netzwerkstörungen der normalen Informationsübermittlung beruht, die durch eine Neuromodulation korrigiert werden können. Wegweisend war die Aufklärung der neuronalen Dysfunktionen der Basalganglien-Kortex-Schleife, die je nach Art der gestörten Nervenzellaktivität zu hypo- oder hyperkinetischen Bewegungsstörungen führen können. Weitere Beispiele für „Rhythmusstörungen“ des Ge- hirns, die ganz ohne morphologischstrukturelle Veränderungen auftreten können, sind der essenzielle Tremor oder bestimmte Epilepsien. Obgleich die elektrochemische Natur der Informationsverarbeitung im Nervensystem lange bekannt ist, haben Elektrotherapien bislang eine Außenseiterrolle in den klinischen Neurowissenschaften gespielt. Auch die THS wird trotz ihrer unbestrittenen Erfolge bei der Parkinson-Krankheit, verschiedenen Tremorformen oder den Dystonien von vielen Neurologen noch als gefährlich und „letzter Ausweg“ bei terminalen Krankheitsstadien gesehen. Dabei ist die Studienevidenz für die THS bei der Parkinson-Krankheit heute derart umfassend, wie für kaum eine der medikamentösen Therapien. Die rasche Entwicklung im Bereich der klinischen Anwendung und Indikationsstellung, aber auch technische Fortentwicklungen auf dem Gebiet der THS haben uns veranlasst, das vorliegende interdisziplinäre Leitthemenheft zu initiieren.

Dabei wird der heutige Kenntnisstand zu Indikation, klinischer Bedeutung und Wirkung der THS bei Morbus Parkinson und hyperkinetischen Bewegungsstörungen zusammengefasst (Erasmi R et al. [1], Reich und Volkmann [2]) und die sich schnell entwickelnde Ergebnislage von ersten Studien zu psychiatrischen Erkrankungen diskutiert (Schläpfer [3], Bartsch und Kuhn [4]). Im Weiteren werden besondere neuroethische Implikationen der THS bei psychiatrischen Erkrankungen dargestellt (Bartsch und Kuhn [4]) sowie sich abzeichnende technische Innovationen im Bereich der THS 
und ihre mögliche zukünftige Bedeutung erörtert (Vesper und Slotty [5]).

Es ist bemerkenswert, dass mittlerweile selbst Vertreter der pharmazeutischen Industrie anerkennen, dass Netzwerkstörungen des Gehirns vermutlich gezielter durch „elektroceuticals“ behandelt werden können als durch klassische pharmakologische Ansätze. Hierzu verweisen wir auf das lesenswerte Editorial von Kristoffer Famm (GlaxoSmithKline) und Kollegen im Aprilheft von Nature [6]. Wir hoffen, dass unser Leitthemenheft dazu beitragen kann, unbegründete Ängste und Vorbehalte gegenüber der THS abzubauen. Auch wir sind der festen Meinung, dass in naher Zukunft der „Hirnschrittmacher" für den Neurologen oder Psychiater so selbstverständlich zum Behandlungsspektrum gehören dürfte, wie der Herzschrittmacher für den Kardiologen.
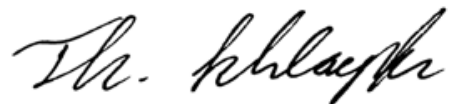

Prof. Dr. Thomas Schläpfer

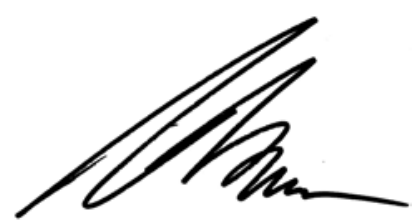

Prof. Dr. Jens Volkmann

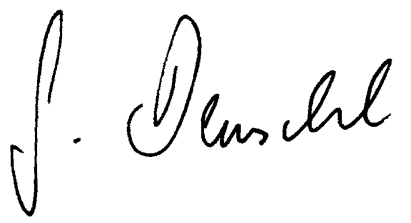

Prof. Dr. Günther Deuschl

\section{Korrespondenzadresse}

\section{Prof. Dr. T. Schläpfer}

Klinik für Psychiatrie und Psychotherapie,

Universitätsklinikum Bonn

Sigmund-Freud-Str. 25, 53105 Bonn

schlaepf@jhmi.edu
Interessenkonflikt. T. Schläpfer erhielt limitierte Unterstützung zur Durchführung von Investigator-initiierten Studien von der Firma Medtronic, einem Hersteller von THS-Geräten. J. Volkmann: Beratertätigkeit und Vorträge mit Honorarzahlung durch die Firmen Medtronic und Boston Scientific. Honorar für Vorträge der Firma St. Jude. Forschungsunterstützung durch Medtronic und Boston Scientific. (Alle Hersteller von Systemen zur tiefen Hirnstimulation.) G. Deusch/ hat Vortragshonorare von Desitin, UCB und Medtronic sowie Buchhonorare von Thieme und Elsevier erhalten. Er hat Beratertätigkeit für Sapiens, Medtronic, TEVA und Britannica erbracht und Forschungsunterstützung von DFG, BMBF und Medtronic erhalten.

\section{Literatur}

1. Erasmi R, Deuschl G, Witt K (2014) Tiefe Hirnstimulation bei Morbus Parkinson: Wann und für wen? Nervenarzt DOI s00115-013-3876-7

2. Reich MM, Volkmann J (2014) Tiefe Hirnstimulation bei hyperkinetischen Bewegungsstörungen. Nervenarzt DOI s00115-013-3877-6

3. Schläpfer TE (2014) Tiefe Hirnstimulation als mögliche Alternative bei therapieresistenten Depressionen. DOI s00115-013-3878-5

4. Bartsch C, Kuhn J (2014) Tiefe Hirnstimulation be Sucht, Anorexie und Zwang. Rationale, klinische Ergebnisse und ethische Implikationen. Nervenarzt DOI s00115-013-3879-4

5. Vesper J, Slotty PJ (2014) Technische Innovationen in der tiefen Hirnstimulation. Nervenarzt DOI s00115-013-3882-9

6. Famm K, Litt B, Tracey KJ et al (2013) Drug discovery: a jump-start for electroceuticals. Nature 496:159-16

\section{Neues Wirkprinzip für Kaliumkanäle entdeckt}

Nervenzellen übertragen Informationen mithilfe von für Kaliumionen durchlässigen Kanälen. Sind diese Kanäle defekt, wird dies mit neuronalen Erkrankungen wie Epilepsie und Depression in Verbindung gebracht. Eine Forschergruppe um Henning Stahlberg vom Biozentrum der Universität Basel ermittelte nun erstmals die vollständige 3-D-Struktur eines Kaliumkanals des Typs HCN. Daraus konnten die Forscher Rückschlüsse über den Wirkungsmechanismus gewinnen.

Kaliumkanäle bilden eine Pore mit einem Filter, der nur für Kaliumionen durchlässig ist und durch das Signalmolekül cAMP gesteuert wird. Bisher ging man davon aus, dass sich die Pore öffnen und schliessen kann und so den Fluss der Kaliumionen reguliert. Stahlbergs Team fand nun Anhaltspunkte für ein anderes Wirkprinzip. Die Forscher rekonstruierten mithilfe von Kristallisationstechniken und der Elektronenmikroskopie die intakte 3-D-Struktur des bakteriellen Kaliumkanals in seiner natürlichen Umgebung in An- und Abwesenheit von CAMP.

So konnten sie feststellen, dass die Pore entgegen der bisherigen Meinung immer geöffnet ist. Die neuen strukturellen Details ermöglichten den Forschern, die Funktionsweise dieser Kanäle aus einem anderen Blickwinkel zu betrachten.

Signalgesteuerte Kaliumkanäle werden auch als «Schrittmacher-Kanäle» bezeichnet. Sie steuern den Herzrhythmus sowie die rhythmische Erregbarkeit von Neuronen. Das genaue Verständnis des Wirkmechanismus ist daher die Grundlage für die Entwicklung spezifischer Arzneistoffe zur Behandlung von Epilepsie oder Herzrhythmusstörungen.

Quelle: Julia Kowal, Mohamed Chami, Paul Baumgartner et al. (2014)Ligand-induced structural changes in the cyclic nucleotidemodulated potassium channel MloK1. Nature Communications, Published Online 28 January 2014, DOI: 10.1038/ ncomms4106 